\title{
En pacientes jóvenes, el uso de finasteride podría asociarse con mayor riesgo de ansiedad, depresión y eventos adversos relacionados con el suicidio
}

Finasteride use in young patients may be associated with an increased risk of depression, anxiety and suicide-related adverse events

\section{Comentado de:}

Nguyen D-D, et al. JAMA Dermatol. 2021;157(1):35-42. doi: 10.1001/jamadermatol.2020.3385. PMID: $33175100^{1}$

\section{Objetivo}

Investigar si el uso de finasteride se asocia a mayor incidencia de ideación o conductas suicidas, y/o a síntomas de ansiedad y/o depresión.

\section{Diseño}

Estudio de farmacovigilancia de casos y no casos.

\section{Pacientes e intervención}

Los datos fueron extraídos de la base de datos de informes de seguridad de eventos adversos de casos individuales de la Organización Mundial de la Salud (VigiBase), que incluye información de 153 países, desde 1967 a 2019. Los casos fueron obtenidos de informes espontáneos de pacientes adultos que habían recibido finasteride en cualquier dosis, para cualquier indicación y que reportaron cualquier efecto adverso. El grupo control fue construido con los reportes de eventos adversos de medicamentos distintos a finasteride documentados en la misma base de datos.

\section{Medición de resultados principales}

El desenlace primario fue la notificación de cualquier evento adverso relacionado con el suicidio, como la ideación, el intento y el suicidio consumado (desenlace denominado suicidalidad) y el secundario, el reporte de depresión o ansiedad.

Los autores analizaron si en los paciente que usaban finasteride los resultados de interés fueron informados con más frecuencia de lo que se esperaría sólo por azar respecto del resto de los fármacos de VigiBase. Este análisis, denominado de desproporcionalidad, es uno de los métodos de tipo cuantitativo para la detección de señales de seguridad empleado en la farmacovigilancia o etapa de comercialización de un fármaco. Combinaron estrategias de análisis estadístico frecuentista y bayesiano, estimando el índice de probabilidad de reportes (Reporting Odds Ratio $[R O R]$ ) y su intervalo de confianza (IC) del $95 \%$ sólo cuando el valor del estimador de Bayes empírico (EBE) alcanzaba el percentilo 5 para todos los eventos adversos de interés. Además, realizaron un análisis de sensibilidad estratificando a los pacientes en cuatro categorías: 1 ) por el motivo de la prescripción (hipertrofia prostática benigna [HPB] y alopecia) y la edad ( $<45$ y $\geq 45$ años); 2) fármacos con diferentes mecanismos de acción pero utilizados para indicaciones similares (minoxidil para alopecia y tamsulosina para HPB); 3) fármacos con un mecanismo de acción y un perfil de efectos adversos similares (dutasterida); 4) informes de suicidio antes y después de 2012 (el año en el que fue publicado y publicitado el primer estudio clínico que reportó una asociación entre el suicidio y el uso de finasteride).

El Consejo Internacional de Organizaciones de Ciencias Médicas (CIOMS) define como "señal de seguridad.a aquella infor- mación que surge de una o múltiples fuentes (incluyendo observaciones y experimentos), que sugiere una potencial asociación causal nueva o un nuevo aspecto de una asociación conocida previamente, entre una intervención y un evento o conjunto de eventos relacionados (tanto efectos adversos como beneficiosos) que se juzga como suficientemente probable como para justificar una acción verificadora. Por otro lado, el ROR es la razón entre las probabilidades de encontrar el término de un evento adverso entre todos los reportes de caso que mencionan un fármaco en particular y la de encontrar el mismo término del evento adverso entre todos los demás reportes de caso de la base de datos que no mencionan a ese fármaco ${ }^{2}$.

\section{Resultados principales}

Fueron identificados 356 casos de suicidio y 2.926 eventos adversos psicológicos asociados con el uso de finasteride, para un total de 3.282 casos. En el análisis de sensibilidad estratificado por edad e indicación fue observada una señal de desproporcionalidad significativa luego de 2012 para la notificación suicidalidad en términos globales (ideación, intento o concreción de suicio) asociada al uso de finasteride entre los pacientes menores de 45 años (ROR 3,47; IC $95 \% 2,90$ a 4,15) y en aquellos con alopecia (ROR 2,06; IC $95 \% 1,81$ a 2,34); no así entre los de 45 o más años o con HPB. Sin embargo, no fue documentada una desproporcionalidad signiticativa en el reporte de suicidio consumado, ni constatada una asociación entre la dosis y la respuesta. Esta señal no fue observada en ninguno de los desenlaces de interés en los análisis de sensibilidad usando tamsulosina, minoxidil y dutasteride. Esta desproporcionalidad no había ocurrido en los reportes anteriores a 2012, lo que podría sugerir la posibilidad de la presencia de un sesgo de notificación.

\section{Conclusiones}

En este estudio de farmacovigilancia se encontraron índices de probabilidad de notificación significativos de cualquier evento adverso relacionado con el suicidio y de eventos adversos psicológicos asociados con el uso de finasteride en pacientes menores de 45 años que lo usaron para el tratamiento de la alopecia. Estas señales de desproporcionalidad de eventos adversos podrían deberse a un mayor estímulo para reportar estos efectos (sesgo de notificación) y/o a una mayor vulnerabilidad de los pacientes jóvenes a los efectos adversos del finasteride.

\footnotetext{
Conflicto de interés de los autores/Fuentes de financiamiento : Financiado por la Agencia de Defensa de la Salud y del Instituto de Urología Vattikuti, EE.UU. Los patrocinadores no tuvieron ningún rol en el diseño y la realización del estudio, la recopilación, gestión, análisis e interpretación de los datos, la preparación, revisión o aprobación del manuscrito y/o la decisión de publicarlo. Uno de sus autores recibió honorarios personales de Astellas Pharma US, Bayer AG, Janssen Pharmaceutica e Intuitive Surgical, no relacionados con este manuscrito.
} 


\section{Comentario}

El finasteride, medicamento indicado para el tratamiento tanto de la alopecia androgénica como de la hiperplasia prostática benigna (HPB) ${ }^{3,4}$, despertó preocupaciones en el último tiempo en la comunidad médica debido a diferentes reportes que asociaron su indicación con la presencia de tendencias suicidas y eventos adversos psicológicos en sus consumidores ${ }^{5-7}$. Estas preocupaciones llevaron incluso a la creación de organizaciones sin fines de lucro como la Fundación del Síndrome Post-finasteride, que acompaña a las personas y familias que padecen una serie de reacciones adversas sexuales, neurológicas y físicas persistentes en pacientes que han tomado finasteride. Asimismo, estos reportes llevaron a que múltiples autoridades gubernamentales de diversos países emitieran alertas sobre esta droga y a la realización de investigaciones para comprender esta problemática.

Tras el análisis realizado, los autores documentan la existencia de una señal de desproporcionalidad en la suicidalidad en personas menores de 45 años para el tratamiento de la alopecia androgénica. Sin embargo, la presencia de posibles sesgos de confusión por indicación y de notificación estimulada comprometen la interpretación de los resultados descritos.

Por otro lado, la validez externa podría verse comprometida debido a la presencia de factores de confusión cointervinientes no tenidos en cuenta en el diseño de la investigación y que podrían explicar los fenómenos de suicidio y los eventos adversos psicológicos, como p. ej. el apoyo social y el nivel socioeconómico, entre otros; las dificultades inherentes al sistema de registro de la información que podrían ocasionar que varios eventos adversos no hayan sido notificados a las autoridades nacionales para su inclusión en VigiBase (limitación que se ve mitigada por la amplitud de la recopilación de datos de 153 países y el gran volumen de informes); el uso concomitante de drogas psiquiátricas que podrían explicar los desenlaces documentados; y la falta de documentación de la duración de la exposición al finasteride.

Es necesario tener en cuenta que los estudios de monitoreo de reacciones adversas medicamentosas (RAM) resultan esenciales para evaluar la seguridad de los fármacos y tienen la particularidad de ser relativamente simples y económicos. Sin embargo, aún contamos con sistemas deficientes de notificación de RAM, por lo que la calidad de la notificación puede ser variable y muchas veces insuficiente, así como estar influenciada por parámetros que dependen del informador, los fármacos, la reacción en sí o el tiempo. Por lo tanto, una señal de desproporcionalidad puede ser el resultado de un aumento real en la incidencia de la reacción adversa o un aumento artificial ${ }^{8,9}$. Es importante recordar que una señal de seguridad en análisis de farmacovigilancia no constituye en sí misma una prueba de asociación causal, sino que indica la necesidad de emprender investigaciones que permitan concluir o descartar que el medicamento sea el causante del evento. Por todos estos motivos, aún se necesitan estudios futuros para estimar mejor la posible asociación entre las variables de interés.

\section{Conclusiones del comentador}

El presente estudio de farmacovigilancia de casos y no casos reportó la existencia de informes desproporcionados de suicidio y eventos adversos psicológicos con respecto a lo esperado entre usuarios jóvenes de finasteride que utilizan esta terapia para la alopecia, lo que constituye una señal de seguridad que justifican la realización de investigaciones que tengan como objetivo confirmar la existencia de una asociación causal y los posibles sesgos que pueden llevar a la confusión de esta asociación.

A pesar de sus defectos, el análisis de las bases de datos de farmacovigilancia sigue siendo una piedra angular para el estudio de las reacciones adversas a los medicamentos.

Eros Miguel Angeletti [ Hospital Italiano de Buenos Aires, Servicio de Medicina Familiar y Comunitaria. eros.angeletti@hospitalitaliano.org.ar ]

Angeletti EM. En pacientes jóvenes, el uso de finasteride podría asociarse con mayor riesgo de ansiedad, depresión y eventos adversos relacionados con el suicidio. Evid Actual Pract Ambul. 2021;24(4):e002152. Available from: https://dx.doi.org/10.51987/EVIDENCIA.V25I1.6982. Comentado de: Nguyen D-D, et al. Investigation of Suicidality and Psychological Adverse Events in Patients Treated With Finasteride. JAMA Dermatol. 2021;157(1):35-42. doi: 10.1001/jamadermatol.2020.3385. PMID: 33175100

\section{Referencias}

1. Nguyen DD, Marchese M, Cone EB, et al. Investigation of Suicidality and Psychological Adverse Events in Patients Treated With Finasteride. AMA Dermatol. 2021;157(1):35-42. Available from: 10.1001/jamadermatol.2020.3385.

2. Practical Aspects of Signal Detection in Pharmacovigilance: Report of CIOMS Working Group VIII. Geneva: Council for International Organizations of Medical Sciences (CIOMS); 2010. Available from: https://cioms.ch/publications/product/practical-aspects-of-signal-detection-in-pharmacovigilancereport-of-cioms-working-group-viii/.

3. Blumeyer A, Tosti A, Messenger A, et al. Evidence-based (S3) guideline for the treatment of androgenetic alopecia in women and in men. J Dtsch Dermatol Ges. 2011;9(6):1-57. Available from: 10.1111/j.1610-0379.2011.07802.x.

4. Oelke M, Bachmann A, Descazeaud A, et al. European Association of Urology. EAU guidelines on the treatment and follow-up of non-neurogenic male lower urinary tract symptoms including benign prostatic obstruction. Eur Urol. 2013;64(1):118-140. Available from: 10.1016/j.eururo.2013.03.004.

5. Levine D. Court let Merck hide secrets about a popular drug's risks; 2019. Available from: https://www.reuters.com/investigates/special-report/usacourts-secrecy-propecia/.

6. Waters J. The drug that banishes baldness can ruin a man's love life: hair loss drug finasteride is used by millions, but men say it has devastating side-effects such IMPOTENCE that can last years after you've stopped taking it.; 2019. Available from: https://www.dailymail.co.uk/health/article7274057/How-baldness-drug-ruin-mans-love-life-Finasteride-devastating-effects.html.

7. Castillo M. Study of ex-Propecia users with sexual side effects finds increased risk for depression, suicidal thoughts; 2012 . Available from: https: //www.cbsnews.com/news/study-of-ex-propecia-users-with-sexual-side-effects-finds-increased-risk-for-depression-suicidal-thoughts/. 
8. Papale R, Schiaffino S. Detección de señales: Manual de buenas prácticas de farmacovigilancia edición latinoamérica. 1st ed. Ciudad Autónoma de Buenos Aires: Ediciones Farmacológicas; 2018. Available from: https://isoponline.org/wp-content/uploads/2018/10/FVG_II_digital_conHipervinculos.pdf.

9. Organización Mundial de la Salud. Señales en farmacovigilancia para las américas. Red Panamericana de Armonización de la Reglamentación Farmacéutica (Red PARF); 2016. Available from: https://www.paho.org/hq/dmdocuments/2016/senales-farmacovigilancia-10-16.pdf. 